\title{
Genome distance between regulatory elements of growth-related genes may determine morpho-physiological traits in mammals
}

\author{
Dmitriy Romanov \\ Academy of biology and biotechnologies \\ Southern federal university \\ Rostov-on-Don, Russia \\ rdme@yandex.ru
}

\author{
Tatiana Shkurat \\ Academy of biology and biotechnologies \\ Southern federal university \\ Rostov-on-Don, Russia \\ tshkurat@yandex.ru
}

\begin{abstract}
The problem of growth regulation in mammals is one of the long-standing mysteries in biology.

We found significant correlation between morphophysiological traits and genome distances between conserved elements in neighborhoods of growth-related genes Mycn, Plagl1 and $E z h 2$ in mammals. Given these conserved elements may be regulatory as well, we propose that genome distance between them may evolutionary modulate gene expression of these genes and eventually affects phenotype. It opens intriguing prospect to control morpho-physiological traits of mammals (i.e. adult body mass or longevity) just simply editing genome distance between these elements.
\end{abstract}

Index Terms - growth regulation, conserved elements, genome distance, morpho-physiological traits, mammals

\section{MOTIVATION AND AIM}

\section{Motivation}

The problem of growth regulation in mammals is one of the long-standing mysteries in biology $[1,2]$.

We have previously revealed strong correlation between morpho-physiological traits and some inter-conserved element distances in the neighborhoods of Mycn, Plagll and $E z h 2$ genes [3]. We propose that genome distance between regulatory elements of the growth-related genes may modulate gene expression of this genes and eventually affects phenotype.

Aim

Many cis-regulatory elements being conserved elements as well, the aim of this research was to study the relationships between morpho-physiological traits of mammals and genome distances between the conserved elements in the neighborhoods of the Mycn, Plagll and Ezh2 genes.

\section{METHODS}

\section{Correlation analysis}

All available genome sequences of \pm 50000 b.p. neighborhoods of Mycn, Plagll and Ezh2 genes of mammals (about 130 species) were obtained.
Genome elements, preserved in each neighborhood of the gene, were considered to be conserved. Each conserved element was assigned a name, i.e. $\operatorname{MYCN}(-6893)$, where MYCN denotes gene and -6893 - genome distance in b.p. between gene start and the middle of the element.

Correlation analysis of the relationships between interconserved element distances in the neighborhoods of the Mycn, Plagll and Ezh2 genes and morpho-physiological traits of mammals was carried out.

Adult body mass, adult body length, age of maturity and lifespan were assessed. The trait values were obtained from the PanTHERIA and AnAGE databases $[4,5]$.

The relationships with the Spearman's correlation coefficient greater than 0.4 and the $p$-value less than 0.05 were considered to be statistically significant. The problem of multiple comparisons was addressed by multiplying the resulting $p$-value by the number of comparisons according to the Bonferroni correction.

\section{Gene Ontology enrichment analysis}

The BLAST search for homologs of the conserved elements in the human genome was carried out. The set of genes in the \pm 50000 b.p. neighborhood of the homologs was obtained. Gene Ontology enrichment analysis of this set of genes was made with the help of Panther classification system [6]

\section{RESULTS}

\section{Correlation}

The correlations revealed in this study (Table I) are generally conform to those obtained previously on the set of 36 mammals [3]. Nevertheless, correlations are weak in spite of $p$-values being very small. It may be due to a fuzzy nature of morpho-physiological trait data. Strong correlation was revealed between EZH2(-8314)-EZH2(196) genome distance and age of maturity. Semilog plots of the inter-element distances and morphophysiological traits for the pairs of the topmost correlated elements are given in Fig. 1. 
TABLE I - CORRELATION BETWEEN THE INTER-CONSERVED ELEMENT DISTANCES AND MORPHO-PHYSIOLOGICAL TRAITS OF MAMMALS

\begin{tabular}{|c|c|c|c|c|c|}
\hline Corr. coeff. & $\mathrm{P}$-value & $\begin{array}{l}\text { Bonferroni corrected } p \text { - } \\
\text { value }\end{array}$ & Element 1 & Element 2 & Trait \\
\hline 0.53 & 4.79E-10 & 4.79E-08 & PLAGL1(79389)* & PLAGL1(112171) & Adult body \\
\hline-0.41 & $1.38 \mathrm{E}-05$ & $1.38 \mathrm{E}-03$ & MYCN(-6893) & $\operatorname{MYCN}(74)$ & mass \\
\hline-0.40 & $2.08 \mathrm{E}-05$ & $2.08 \mathrm{E}-03$ & MYCN(-6893) & MYCN(1273) & \\
\hline 0.54 & $5.73 \mathrm{E}-09$ & $5.73 \mathrm{E}-07$ & PLAGL1(79389) & PLAGL1(112171) & Adult body \\
\hline-0.44 & $1.90 \mathrm{E}-05$ & $1.90 \mathrm{E}-03$ & MYCN(-25368) & MYCN(1273) & length \\
\hline-0.41 & $7.06 \mathrm{E}-05$ & 7.06E-03 & MYCN(-6893) & MYCN(74) & \\
\hline 0.40 & $1.95 \mathrm{E}-05$ & $1.95 \mathrm{E}-03$ & PLAGL1(79389) & PLAGL1(112171) & Max \\
\hline-0.51 & $1.24 \mathrm{E}-04$ & $1.24 \mathrm{E}-02$ & EZH2(-8314) & EZH2(194) & longevity \\
\hline-0.75 & $1.14 \mathrm{E}-09$ & $1.14 \mathrm{E}-07$ & EZH2(-8314) & EZH2(194) & Sexual \\
\hline-0.54 & $1.08 \mathrm{E}-06$ & $1.08 \mathrm{E}-04$ & EZH2(-8314) & EZH2(1627) & maturity age \\
\hline
\end{tabular}

*Elements, which overlap gene promoters, are highlighted in bold
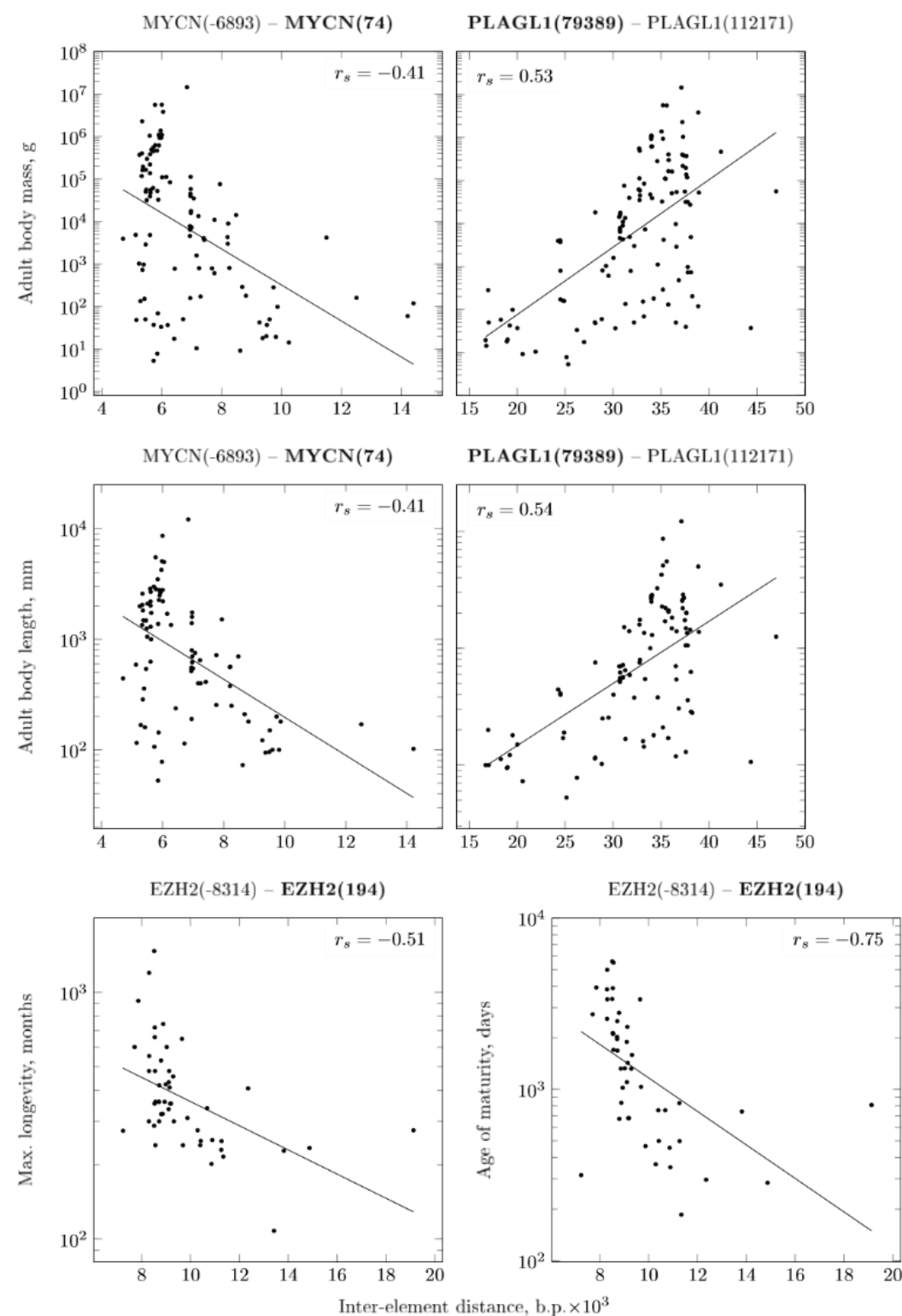

Fig. 1. Semilog plots of the inter-conserved element distances and morphophysiological trait for the pairs of the topmost correlated conserved elements in the neighborhoods of the Mycn, Plagl1 and Ezh2 genes. Elements, which overlap gene promoters, are highlighted in bold. Spearman's rank correlation coefficent is provided for every plot with the line of best fit 
In each plot dots appear to line up along the straight line suggesting that morpho-physiological traits depend exponentially on the inter-element distance.

\section{Gene Ontology enrichment analysis}

The BLAST search for homologs of the topmost correlated conserved elements was made.

Surprisingly, the promoter overlapping elements MYCN(74), PLAGL1(112171) and EZH2(194) were presented in a single copy in the genome, whereas the corresponding elements of the topmost correlated pair - MYCN(-6893), PLAGL1(79389) and EZH2(-8314) - were scattered across the genome.

It suggests that elements $M Y C N(74)$, PLAGL1(112171) and EZH2(194) may be regarded as gene-specific regulatory elements, whereas elements MYCN(-6893), PLAGL1(79389) and EZH2(-8314) may represent universal regulatory elements, capable to control many neighboring genes.

Gene Ontology enrichment analysis of the set of genes in the \pm 50000 b.p. neighborhood of homologs of elements MYCN(6893), PLAGL1(79389) and EZH2(8314) was made.

It was found that $A C V R 1 B$ and $A C V R L 1$ genes in the vicinity of homolog of element EZH2(-8314) are significantly overrepresent category response to growth factor, GO:0070848, $p=0.046$.

Thus, it suggests that element EZH2(-8314) may do be a regulatory sequence and control growth-related genes $E Z H 2, A C V R I B$ and $A C V R L 1$ along with other genes.

\section{ACKNOWLEDGMENT}

The study was performed on the equipment of the Center of collective use "High technologies" (Southern federal university, Rostov-on-Don, Russia) the framework of the state task of Russian Ministry of education and science "Biochemical and molecular genetic studies of pathological processes mechanisms associated with socially significant diseases", № BAZ0110/20-5-14AB.

\section{REFERENCES}

[1] J. C. Lui, J. Baron, Mechanisms limiting body growth in mammals, Endocr. Rev. 32 (3) (2011) 422-440.

[2] A. Delaney et al. Evolutionary conservation and modulation of a juvenile growth-regulating genetic program, J. Mol. Endocrinol. 52 (3) (2014) 269-277.

[3] Genome distance between conserved elements in neighborhoods of growth-regulating genes is correlated with morpho-physiological traits in mammals / D. E. Romanov, E. V. Butenko, G. B. Bakhtadze, T. P. Shkurat // Gene Reports. 2019. - Vol. 17.

[4] Jones, K.E. et al. (2009) PanTHERIA: a specieslevel database of life history, ecology, and geography of extant and recently extinct mammals. Ecology 90, 2648.

[5] Tacutu, R et al. (2018) Human Ageing Genomic Resources: new and updated databases. Nucleic Acids Res., 46, D1:D1083-D1090.

[6] Mi, H, Muruganujan, A, Thomas, PD (2013). PANTHER in 2013: modeling the evolution of gene function, and other gene attributes, in the context of phylogenetic trees. Nucleic Acids Res., 41, Database issue:D377-86. 